\title{
INVARIANT SUBSPACES OF OPERATORS RELATED TO THE UNILATERAL SHIFT
}

\author{
Dedicated to the memory of Hanna Neumann
}

S. R. CARADUS

(Received 11 May 1972)

Communicated by J. P. O. Silberstein

\section{Introduction}

Among all non-self-adjoint operators, the shift has a special place in questions relating to invariant subspaces. It is therefore natural to attempt to use this fact to study other operators related in some way to the shift. Examples of this procedure are available in the work of Freeman [3] and Lim [5] where perturbations of the shift are studied.

In this paper, some modest contributions are made to the invariant subspace problem for certain classes of operators.

Let $H$ be a separable Hilbert space with orthonormal basis $\left\{e_{k}\right\}_{1}^{\infty}$. Then write $S$ to denote the shift operator $S: e_{k} \rightarrow e_{k+1}, k=1,2, \cdots$. Since $S$ is an isometry, we know that $S^{*} S=I$.

Now let $T$ be a bounded linear operator. We wish to state conditions sufficient to ensure that $T$ is intransitive, i.e. that $T$ has a non-trivial closed invariant subspace. For this purpose, it is no loss of generality to assume that $\|T\|<1$.

Consider the mapping defined in $\mathrm{H}$ as follows:

$$
x \rightarrow \sum_{k=1}^{\infty}\left(T^{k-1} x, e_{1}\right) e_{k} .
$$

It is easy to verify that this map is well defined and gives a bounded linear operator on $H$. Let $H_{T}$ denote the range of this operator. In view of the theorem which follows, it is useful to investigate whether $H_{T}$ is invariant under $T$. If this were the case, a simple calculation shows that this implies that, for each $x \in H$, there must exist $\bar{x}$ in $H$ such that the following conditions are satisfied:

$$
\left(T^{k-1} \bar{x}, e_{1}\right)=\sum_{p=1}^{\infty}\left(T^{p-1} x, e_{1}\right)\left(T e_{p}, e_{k}\right) \quad k=1,2,3, \cdots
$$


Since it seems highly unlikely that such a condition would be satisfied, it is reasonable to conclude that in general $H_{T}$ is not invariant under $T$.

THEOREM. If $H_{T}$ is not dense, then $T$ has a non-trivial closed invariant subspace.

Proof. Write $P$ to denote the projection $x \rightarrow\left(x, e_{1}\right) e_{1}$ so that clearly $S^{*} P=0$. Define an operator $\Phi_{T}$ by the equation

$$
\Phi_{T}=\sum_{k=0}^{\infty} S^{k} P T^{k}
$$

The series obviously converges in the uniform operator topology; in fact, $\Phi_{T}$ is compact since $P$ is one-dimensional. Moreover, it is easy to verify that

$$
S^{*} \Phi_{T}=\Phi_{T} T
$$

so that the range of $\Phi_{T}$ is invariant under $S^{*}$ (briefly, "*-invariant"). Now we observe that the range of $\Phi_{T}$ is exactly $H_{T}$. With our assumption that $H_{T}$ is not dense, we will show the existence of a ${ }^{*}$-invariant subspace $H_{0}$ of $H_{T}$ such that (0) $\neq \bar{H}_{0} \varsubsetneqq \bar{H}_{T}$. To do this, we will work in analytic terms. Let $D$ denote the open unit disc in the complex plane and let $H^{2}$ denote the usual Hardy class on $D$. Then, since $H_{T}$ is not dense, $H_{T}^{\frac{1}{2}}$ is a closed non-trivial subspace of $H^{2}$ which is invariant under $S$. Hence, as Beurling showed, [1], $H_{T}^{\perp}=\theta H^{2}$ for some inner function $\theta$. Let $z_{0}$ be any zero of $\theta$. Define $H_{0}=\left(S^{*}-z_{0}\right) H_{T}$; obviously $H_{0}$ is *-invariant and $(0) \neq H_{0} \subseteq H_{T}$. To show that the inclusion $\bar{H}_{0} \subset \bar{H}_{T}$ is proper, it suffices to show that $H_{T}^{\perp} \neq H_{0}^{\perp}$. Let $v$ be an element of $H^{2}$ such that $v\left(z_{0}\right) \neq 0$ and take $h(z)=\theta(z) v(z) /\left(z-z_{0}\right)$. Then $h \in H^{2}$ and, if $w_{0} \in H_{0}$, we have

$$
\begin{aligned}
\left(h, w_{0}\right) & =\left(\frac{\theta(z) v(z)}{z-z_{0}},\left(S^{*}-\bar{z}_{0}\right) w\right) \text { for some } w \in H_{T} \\
& =(\theta(z) v(z), w(z))=0 \text { since } \theta v \in H_{T}^{\perp} .
\end{aligned}
$$

Hence $h \in H_{0}^{\perp}$. But $h \notin H_{T}^{\perp}$ for otherwise, we would have $h=\theta h^{\prime}$ for some $h^{\prime} \in H^{2}$. This would imply

$$
\theta h^{\prime}=\frac{\theta v}{z-z_{0}}
$$

i.e.

$$
h^{\prime}=\frac{v}{z-z_{0}} \text {. }
$$

But $\frac{v(z)}{z-z_{0}}$ cannot be a function in $H^{2}$. Hence $H_{T}^{\perp} \neq H_{0}^{1}$ so that $H_{0}$ has the required properties.

Finally, consider equation (1). Evidently, the kernel of $\Phi_{T}$ is invariant under $T$ so we need only consider the case where the kernel of $\Phi_{T}$ is (0). Let $\tilde{H}=\Phi_{T}^{-1} H_{0}$. 
We will show that $\tilde{H}$ is nondense and invariant under $T$. Firstly, if $\tilde{H}$ were dense, then every $x$ in $H$ could be written

$$
x=\lim \Phi_{T}^{-1} h_{n} \text { for some sequence }\left\{h_{n}\right\} \subset H_{0} .
$$

But then

$$
\begin{aligned}
\Phi_{T} x & =\lim h_{n} \\
\text { i.e. } H_{T} & \subseteq \bar{H}_{0}
\end{aligned}
$$

contrary to the known properties of $H_{0}$. Finally $\tilde{H}$ is $T$-invariant, for if $x \in \Phi_{T}^{-1} H_{0}$, then $\Phi_{T} x=h_{0}$ for some $h_{0} \in H_{0}$. Hence $\Phi_{T} T x=S^{*} \Phi_{T} x=S^{*} h_{0} \in H_{0}$ i.e. $T x \in \tilde{H}$. Thus the closure of $\tilde{H}$ is non-trivial closed invariant subspace for $T$.

REMARK. A bounded linear operator with trivial kernel and dense range has been called a "quasi-affinity" [6]. If $A$ and $B$ are bounded linear operators on $H$ and there exists a quasi-affinity $Q$ such that $Q A=B Q$, then $A$ is said to be a "quasi-affine" transformation of $B$. Theorem 1, therefore, implies that either $T$ has a non-trivial closed invariant subspace or $T$ is a quasi-affine transformation of $S^{*}$. Sz-Nagy and Foias [6] have proved a stronger result: if $T^{*}$ has a cyclic vector, then $T$ is a quasi-affine transformation of $S^{*}$. However, in their case, the operator which implements the transformation is difficult to express in terms of the given Hilbert space.

COROLLARY. $H_{T}$ is non-dense if and only if, for some non zero $\left\{x_{k}\right\}_{0}^{\infty}$ in $l^{2}$, we have $\sum_{k=0}^{\infty} \alpha_{k} T^{* k} e_{1}=0$.

PRoof. A vector $y=\sum_{1}^{\infty} \alpha_{k-1} e_{k}$ is orthogonal to $H_{T}$ if

$$
\begin{aligned}
\sum_{1}^{\infty}\left(T^{k-1} x, e_{1}\right) \alpha_{k-1} & =0 \text { for all } x \text { in } H \\
\text { i.e. }\left(x, \sum_{1}^{\infty} \bar{\alpha}_{k-1} T^{* k-1} e_{1}\right) & =0 \text { for all } x \text { in } H \\
\text { i.e. } \sum_{0}^{\infty} \bar{\alpha}_{k} T^{* k} e_{1} & =0 .
\end{aligned}
$$

REMARKS. (i) The role of $e_{1}$ in the above corollary is obviously incidental. If $x$ is any unit vector in the kernel of $\sum_{0}^{\infty} \alpha_{k} T^{* k}$ for some $\left\{\alpha_{k}\right\}$ in $l^{2} \backslash\{0\}$, then we can choose the orthonormal basis with $x$ as its first vector.

(ii) Recall that $H^{\infty}$ consists of functions analytic and bounded on $D$. Let $u \in H^{\infty}$. Then we can write a Taylor series for $u$

$$
u(\lambda)=\sum_{0}^{\infty} u_{k} \lambda^{k}
$$

and since $H^{\infty} \subset H^{2}$, we know that $\Sigma_{0}^{\infty}\left|u_{k}\right|^{2}<\infty$. Now recall the important class 
$\mathrm{C}_{0}$ defined by Sz-Nagy and Foias [7]: $C_{0}$ consists of those completely nonunitary contractions $T$ for which there exists a non-zero $u$ in $H^{\infty}$ such that $u(T)=0$. If $T$ is a strict contraction (i.e. $\|T\|<1$ ) then it is possible to define $u(T)$ as $\sum_{0}^{\infty} u_{k} T^{k_{i}}$. In view of these considerations, we see that if $T$ is a strict contraction in $C_{0}$, then $H_{T}$ is non-dense and so $T$ has a non-trivial closed invariant subspace. Again, this is a known result but the methods used here are simple and explicit and, of course, the condition expressed in the corollary above is much weaker than the condition $u(T)=0$.

(iii) Gohberg and Krein [4] p. 316 have defined a sequence of vectors $\left\{g_{k}\right\}$ to be $w$-linearly independent if $\Sigma \alpha_{k} g_{k}=0$ is not possible for $0<\Sigma\left\|\alpha_{k}\right\|^{2}\left\|g_{k}\right\|^{2}<\infty$. It is evident that if $\left\{T^{* k} e_{1}\right\}_{0}^{\infty}$ is $w$-linearly independent then the condition of the corollary above fails.

ACKNowledgement: Thanks are due to my colleague, C. F. Schubert, for useful discussions about this work.

\section{References}

[1] A. Beurling, 'On two problems concerning linear transformations in Hilbert space', Acta Math., 81 (1949), 239-255.

[2] S. R. Caradus, 'Universal operators and invariant subspaces', Proc. Amer. Math. Soc. 23 (1969), 526-527.

[3] J. M. Freeman, 'Perturbations of the shift operator', Trans. Amer. Math. Soc. 114 (1965), 251-260.

[4] I. C. Gohberg and M. G. Krein, Introduction to the theory of linear non self adjoint operators (Translations of Mathematical Monographs, Vol. 18, Amer. Math. Soc., (1969).

[5] S. C. Lin, 'Perturbations of the unilateral shift', Bull. Amer. Math. Soc. 77 (1971), 621-624.

[6] B. Sz-Nagy and C. Foias, 'Vecteurs cycliques et quasi-affinités', Studia Math. 31 (1968), $35-42$.

[7] B. Sz-Nagy and C. Foias, Harmonic Analysis of Operators on Hilbert Space (North-Holland, American Elsevier, 1970).

Queen's University at Kingston

Department of Mathematics

Ontario, Canada 